\title{
Dicotomías y emblematizaciones persistentes en la educación intercultural bilingüe Una lectura desde México
}

Recibido: 10 de abril de 2021 Aceptado: 17 de agosto de 2021

1 Universidad Pedagógica Nacional (UPN), Ajusco, México

(D) https://orcid.org/oooo-0002-7638-4229.

Correo electrónico: gacza_2006@yahoo.com.mx

2 Centro de Investigaciones y Estudios Superiores en Antropología Social (CIESAS), CDMX, México

(D) https://orcid.org/0000-0003-0641-1567

Correo electrónico: julietabriseno@ciesas.edu.mx

\section{Resumen}

El texto busca aportar al debate sobre lo que en América Latina se denomina Educación Intercultural Bilingüe (EIB) dirigida a las comunidades indígenas. Nos proponemos visibilizar la emblematización de nociones centrales como lengua y cultura a partir de posiciones dicotómicas presentes en las políticas educativas interculturales. Nos detenemos en proyectos sostenidos en el bilingüismo que marcan identidades, y en políticas que mantienen a un 'otro' diferente como sujeto de atención por su vulnerabilidad y no como sujeto de derecho. Planteamos un ejercicio de reflexión a partir de nuestras investigaciones de corte etnográfico e incluimos ejemplos referidos a la escolarización de niñas/os y jóvenes indígenas en contextos rurales y urbanos y a experiencias educativas a partir de modelos pedagógicos comunitarios en algunas regiones de México. Desarrollamos estos argumentos bajo una perspectiva antropológica que mira a la escuela como espacio de disputa en el que se relacionan el Estado y diversos actores sociales.

\section{Persistent dichotomies and emblematizations in intercultural bilingual education: a reading from Mexico}

\begin{abstract}
The text seeks to contribute to the debate on what in Latin America is called Intercultural Bilingual Education (IBE), aimed at indigenous communities. We propose to make visible the emblematization of central notions such as language and culture from dichotomous positions present in intercultural educational policies. We focus on projects sustained in bilingualism that mark identities, and on policies that maintain a different 'other' as a subject of attention due
\end{abstract}

\section{Palabras clave}

Educación intercultural; Educación indígena: Bilingüismo; Cultura; Identidad

\section{Key wards}

Intercultural education; Indigenous education; Bilingualism; Culture; Identity 
to their vulnerability, and not as a subject of law. We propose a reflection exercise based on our ethnographic research that include examples referring to the schooling of indigenous children and young people in rural and urban contexts and educational experiences based on community pedagogical models in some regions of Mexico. We develop these arguments from an anthropological perspective that looks at the school as a space of dispute where the State and various social actors interact.

\section{Dicotomias e emblematizações persistentes na educação bi- lingue intercultural: uma leitura do México}

\section{Resumo}

Palavras-chave

Educação intercultural; Educação indígenas; Bilinguismo; Cultura, identidade
O texto contribui para o debate sobre o que na América Latina é chamado de Educação Intercultural Bilíngüe (EIB), voltada para as comunidades indígenas. O objetivo é tornar visível a 'emblematização' de noções centrais como idioma, cultura e processos bilíngues para marcar identidades, expressada em políticas que mantém a diferenciação de um 'outro' colocado como sujeito de atenção devido a sua vulnerabilidade, e não como sujeito de direitos. Propomos um exercício de reflexão baseado em nossas pesquisas etnográficas e com exemplos referentes a processos de escolarização indígena em contextos rurais e urbanos de crianças e jovens indígenas, e bem como modelos pedagógicos comunitários em algumas regiões do México. Analisamos duas categorias dicotômicas utilizadas nas políticas educacionais interculturais e bilingüe que continuam com tais emblematizações. Assumimos uma perspectiva antropológica que considera a escola como uma arena de disputa onde o Estado e vários atores sociais se relacionam, e a escola é sempre um exercício político e pedagógico com múltiplas travessias.

\section{Introducción}

En este texto buscamos debatir algunas de las posiciones arraigadas en las políticas educativas dirigidas a las poblaciones denominadas 'indios y/o indígenas', particularmente a través de lo que en Latinoamérica conocemos como Educación Intercultural Bilingüe (EIB). El mismo término de indígena, conceptualización que proviene de una marca externa puesta por los colonizadores que llegaron a las tierras que se conocen como América y también como Abya Yala, ha sido una base para construir una alteridad (López-Caballero, 2015) que, en términos de Quijano (2000), resulta útil para la perpetuación de una historia profunda de colonialidad y racialización de los pueblos.

Partimos de una reflexión sobre la denominada EIB, que desde la década de 1970 se encuentra presente en la definición de las políticas educativas dirigidas a los pueblos indígenas. Entre las concepciones que subyacen y mantienen continuidad en la postulación de la EIB en clave funcional para el Estado (Tubino, 2004) identificamos una serie de binarismos que marcan dicotomías para abordar el tratamiento de estas propuestas educativas, entre ellas: a) indígena-mestizo o nacional, la cual considera una suerte de homogeneidad 
en ambas partes del binomio, y se invisibiliza así la complejidad de las imbricadas estructuras socioculturales de la región y, al mismo tiempo, se niega la diversidad de formas de vida, epistemologías y ontologías de los pueblos originarios y de las categorías étnicas marcadas desde afuera y que se han establecido para usar estas diferencias (Bonfil, 1991; Pérez Ruiz, 2016; Navia, Czarny y Salinas, 2020); b) conocimientos indígenas/tradicionales-conocimientos científicos: postulación por medio de la que se fortalecen jerarquías a la hora de pensar, por ejemplo, propuestas curriculares, al situar a los primeros como carentes de legitimidad -desde el saber hegemónico occidental-y por eso son excluidos de las instituciones educativas en tanto sistemas válidos de conocimiento (Brayboy y Maughan, 2009; Perez-Ruiz, 2016); c) cultura comunitaria-cultura escolar, como si fuera imposible construir una cultura comunitaria escolar, y como si lo escolar no estuviera permeado por prácticas comunitarias al tiempo que las comunidades se apropian, en distinta medida, también de lo escolar (Jiménez, 2009; Briseño-Roa, 2018); d) rural-urbano: a través de la que se mantiene una imagen tipificada sobre lo indígena ligado a una condición de vida rural y, con ello, alejado del 'desarrollo nacional', que tendría a la ciudad como referencia, con lo cual se invisibiliza a miles de personas y comunidades indígenas que viven ya por generaciones en las urbes (Martínez, 2007; Czarny, 2017).

Esta problematización del campo deja al descubierto la negación de los pueblos y comunidades indígenas a quienes no se les reconoce como sujetos históricos que han enfrentado las transformaciones de procesos culturales complejos, marcados por discriminaciones y racismos, desigualdades e injusticias epistémicas. En esta línea y desde el discurso de una educación intercultural introducida como política pública hemos asistido a un ejercicio que mantiene a un otro diferente por su lengua y cultura y, recuperando a Díaz-Couder (2000), al ubicarlo como sujeto de atención por su vulnerabilidad, y no como sujeto y colectivo de derechos.

A los efectos de visibilizar algunos encubrimientos que se perpetúan en la denominada EIB y que orientan el trabajo educativo desde las políticas de Estado, nos enfocaremos en el análisis de dos dimensiones que funcionan como emblemas y dicotomías, que remiten a: un modo estático y esencialista de concebir a la cultura y la lengua de sujetos/comunidades indígenas para definirlos en sus identidades desde afuera; y la persistencia de propuestas binarias en los enfoques sobre bilingüismo aplicados a lo escolar para comprender realidades multilingües complejas.

La perspectiva metodológica que orienta nuestros trabajos de investigación tiene como referente a la antropología de la educación, que mira la escuela como arena política donde se relaciona el Estado con diversos actores sociales en contextos rurales y urbanos caracterizados por una gran diversidad social, cultural y étnica (Rockwell y González, 2016). En diálogo con esa posición, hacer etnografía en el campo de la educación, en nuestra perspectiva, implica echar a andar un trabajo con las/los sujetos en contextos locales, así como poner atención a los modos en que los actores dan sentido a sus prácticas cotidianas (Rockwell y Anderson-Levitt, 2017), las que se enmarcan en estructuras sociales y de poder. En este texto planteamos un ejercicio de reflexión y análisis a partir de nuestras indagaciones referidas a procesos de escolarización indígena en contextos rurales, urbanos y de migración de niñas/os y de jóvenes indígenas que acceden a la educación superior, así como del acompañamiento a modelos pedagógicos comunitarios indígenas. La posibilidad de contrastar nuestras experiencias de trabajo realizadas con actores en 
escenarios escolares y comunitarios diversos nos permitió preguntarnos, por ejemplo, ¿cómo entienden las/los maestras/os indígenas de algunas regiones de los estados de Chiapas y Oaxaca la denominada EIB y la educación comunitaria? ¿Qué posiciones se identifican en los trabajos escolares a partir de las políticas educativas interculturales bilingües impulsadas desde el Estado o por las propuestas comunitarias a la hora de procurar experiencias educativas que tengan sentido para las/los niñas/os y jóvenes de las comunidades? Bajo estas interrogantes ponemos en la palestra de este escrito algunos ejemplos etnográficos, reconociendo que el trabajo interpretativo que orienta estos haceres de investigación se encuentra atravesado por las desigualdades en distintas dimensiones que marcan las condiciones de lo que se entiende por producir conocimiento en diálogo con otra/o/as/os (Rufer, 2012). Asimismo, consideramos que el análisis de lo educativo y de lo escolar resulta siempre un ejercicio político y pedagógico con múltiples cruces, proceso que se nutre de trabajos de corte socioantropológico realizados por investigadoras/es nativas/os -en tanto partícipes directos de los contextos y las comunidades-, así como por no nativas/os que han observado a las prácticas escolares y comunitarias con la finalidad de aportar al debate sobre la justicia epistémica en procesos escolares.

\section{Rutas divergentes en los discursos multiinterculturales en el campo educativo}

El concepto de interculturalidad para el campo educativo en la región tiene ya vida propia, en particular en el marco de las acciones que desde mediados del siglo pasado vienen impulsando diversos sectores y actores, a la hora de plantear y buscar insertar la escuela del modelo nacional en comunidades indígenas. Desde las primeras propuestas denominadas como Educación Bilingüe Bicultural (EBB), hasta las más recientes de Educación Bilingüe Intercultural (EBI) o Educación Intercultural Bilingüe (EIB), así como bajo otras formas de definir una educación escolar para los pueblos indígenas, el debate se ha complejizado y ha permitido mostrar que no es lo mismo cuando se plantea desde el Estado que cuando lo proponen las mismas organizaciones y agentes sociales. No obstante, la característica polisémica actual de la interculturalidad (Walsh, 2009) ha promovido su uso tanto en los discursos oficiales de la EIB como desde la academia y las organizaciones sociales para acercarse a realidades diversas y complejas.

El discurso de la EIB se incorporó como parte de las agendas y políticas educativas en los Estados nacionales y ello resulta en parte como efecto de las luchas de las organizaciones indígenas que reclaman el reconocimiento de los pueblos y sus derechos a definir sus políticas en el ámbito educativo, así como a regirse por sus propias formas de gobierno y prácticas culturales, sustentado en el Acuerdo 169 de la Organización Internacional de Trabajo en 1989. Esto último también condujo a cambios en las cartas magnas de casi todos los países de la región, a partir de lo cual se inicia un primer nivel de reconocimiento de sus derechos. No es el objetivo de este texto mostrar la diversidad de acciones y elementos que se conjugaron en cada país para plantear la EIB. Más bien nos interesa reconocer que, a más de cincuenta años de la existencia de estas políticas educativas, nos encontramos con posiciones políticas y pedagógicas, así como prácticas escolares diferentes que se denominan interculturales.

En Latinoamérica, la EIB se vincula de modo directo a la educación dirigida principalmente a las comunidades y pueblos denominados 'indígenas'. Esta orientación hacia lo indígena ha girado en las últimas décadas relativamente 
a abrir el debate educativo a diversos sectores que en distinta medida cargan con una marca de diferenciación y alteridad. Es así que la extensión de una perspectiva intercultural se postula para afrodescendientes y, en algunos escenarios, incluye también a sectores denominados vulnerables y a migrantes (Mato, 2018).

La presencia de estas propuestas educativas dirigidas a todas estas diversidades resultó en un discurso políticamente correcto que ha sido impulsado también por los organismos internacionales. Bajo el cobijo de la noción de sociedades multiculturales, a partir de la cual se reconoce a las diversidades y su tipificación en identidad(es), el tema de lo étnico, y con la categoría de 'indígenas', se produce una "emblematización de los signos diacríticos de una supuesta diferencia" (Segato, 2007, p. 64). Siguiendo a la autora, frente al modo de definir y construir la idea de 'una nación' se ha generado una noción simplificada y objetivadora de las llamadas diferencias y alteridades, a través de la cual se niegan los procesos constitutivos en condiciones históricas diversas (Segato, 2007). Lo anterior no solo distorsiona la complejidad de la interseccionalidad que en estas diferencias se pueden combinar -por ejemplo, ser joven, indígena, migrante-, sino que, al mismo tiempo, las diversidades y su tipificación en identidad(es) son parte de la postulación de un multiculturalismo descontextualizado.

Sin embargo, las perspectivas educativas denominadas interculturales bilingües permitieron, por un lado, interpelar al sistema escolar bajo la demanda de derechos para los pueblos indígenas, buscando que las lenguas originarias tuvieran el merecido y necesario lugar para desarrollarse y generar aprendizajes vinculados a sus historias y memorias colectivas (López, 2019). Por otro lado, generar un sentido de la escuela como espacio pedagógico de proyectos propios y construidos desde las comunidades. No obstante, estos giros prometedores por una escolarización con posibilidades emancipatorias han sido cooptados, como lo señala Tubino, por un interculturalismo funcional que sustituyó el discurso de la pobreza estructural por el discurso de la cultura, desplazando el peso que tienen -para comprender las relaciones interculturales- las desigualdades económicas, así como las relaciones de poder (Tubino, 2004). En relación con esto último agregamos también que lo complejo de los discursos por la interculturalidad como supuesto reconocimiento -y más en el marco de lo que se ha denominado multi e interculturalismo neoliberal en su fase posindigenista- es que se otorgan o abren espacios desde el Estado para la participación de los indígenas en áreas diversas. Por ejemplo, en el campo educativo, con la creación de múltiples propuestas denominadas interculturales o afines se avanza, al mismo tiempo, en el despojo territorial y se reducen las condiciones para el ejercicio de justicia para los pueblos (Hernández, Sieder y Sierra, 2013).

En este mismo sentido, el ejercicio analítico requiere diferenciar cuando las luchas comunitarias e indígenas y sus voceros demandan el uso de sus lenguas y la valoración de sus prácticas culturales, como un sitio de reivindicación identitaria, que puede y es usado para una defensa estratégica (Spivak, 2003). En contraste, las políticas interculturales funcionales al Estado se apropian de símbolos y elementos culturales en lo educativo, como lo es por ejemplo incluir unas horas de enseñanza de la lengua como elemento fundamental de la interculturalidad del currículum nacional. 


\section{Notas sobre la Educación intercultural bilingüe como política educativa en México}

En México, desde inicios de la década de 1970, se propone una educación denominada en los inicios Bilingüe Bicultural, y hacia la década de 1990 como Intercultural Bilingüe para las escuelas del nivel de preescolar y primaria para el medio indígena. A partir del año 2000 se crean también desde las políticas de Estado algunas universidades y escuelas normales que se han definido bajo la denominada EIB. No obstante, la castellanización ocupa el lugar central en el contexto de la política educativa bilingüe dirigida a la población indígena y el reconocimiento de la diversidad se mantiene en elementos culturales y lingüísticos determinados.

A partir de la creación, en 1978, de la Dirección General de Educación Indígena (DGEI) -subsistema dentro de la Secretaría de Educación Pública (SEP) y encargada de la educación dirigida a poblaciones indígenas (actualmente llamada Dirección General de Educación Indígena Intercultural y Bilingüe, DGEIIB)-, tanto la presencia como el sentido de las lenguas indígenas y, particularmente, la elaboración de libros de texto gratuitos en estas, comenzaron a tener un lugar en los procesos educativos, aunque ello no ha resuelto, por ejemplo, la alfabetización ni el desarrollo en las lenguas originarias. Las escuelas primarias a cargo del subsistema de DGEIIB comparten la premisa de impulsar un currículum común para toda la educación básica, y para estas escuelas se agrega la perspectiva intercultural y bilingüe. La propuesta actual de bilingüismo se sustenta en el documento Parámetros Curriculares de la Lengua Indígena (DGEI, 2008), con un enfoque de enseñanza basado en las prácticas sociales de la lengua. A la par de la publicación de estos parámetros se incorporó la asignatura de Lengua Indígena como un espacio curricular en las escuelas de la DGEIIB. Sin embargo, esta perspectiva solo contempla los niveles de preescolar y primaria $\left(1^{\circ}\right.$ a $6^{\circ}$ de educación básica o fundamental) y no de secundaria $\left(7^{\circ}\right.$ a $9^{\circ}$ de educación básica). Ello representa dificultades para las niñas y niños hablantes de una lengua indígena como lengua materna, quienes tienen que acceder al dominio del español como lengua de comunicación, de enseñanza y de aprendizaje cuando deciden continuar con la educación secundaria y lo que resta (Czarny y Salinas, 2020). La situación se complejiza aún más en los planes y programas de educación básica vigentes -llamados Aprendizajes Clave para una Educación integral-, en los que se incorporó al currículum de educación preescolar y primaria las asignaturas de Lengua Materna (Español/ Lengua indígena) y Segunda Lengua (Español/Lengua indígena) y Lengua Extranjera (Inglés) (SEP, 2017, 2019).

Sin embargo, la presencia de la niñez y las juventudes indígenas hoy se reconoce en diferentes niveles del sistema educativo, particularmente en la educación básica. Por ejemplo, en las escuelas de preescolar indígena atendidas por la DGEIIB se ubican el 68\% de las niñas y niños indígenas, mientras que en las primarias indígenas, el $54 \%$; un porcentaje importante de esta población se encuentra en las escuelas de preescolar y primarias de tipo general (no de la DGEIIB) $-23 \%$ y $43 \%-$, mientras que en la secundaria, la mayor proporción de jóvenes indígenas se encuentran en telesecundaria (52,4\%), en secundarias técnicas (23,3\%) y en secundarias de tipo general (21,4\%) (UNICEF/INEE, 2018). Esta demarcación entre escuelas de educación indígena y escuelas generales aunque todas conforman el sistema educativo nacional- mantiene la dicotomía de escuelas para indígenas y para no indígenas. No obstante, como lo muestran las cifras anteriores, tanto en zonas urbanas como en las semiurbanas y rurales, el estudiantado indígena está presente, aunque sus adscripciones comunitarias 
son negadas por las instituciones, que tienen el afán de homogeneizar en tanto supuesto sinónimo de igualar. Al mismo tiempo, las y los niños y jóvenes indígenas han desarrollado procesos de mimetismo y ocultamiento identitarios para enfrentar la discriminación sobre sus orígenes étnicos (Czarny, 2017), lo cual potencia la invisibilización en las instituciones.

Los resultados de aprendizaje que el sistema mide dejan siempre en profunda desventaja a estos sectores. Lo anterior remite a que, además del sesgo en la perspectiva de evaluación estandarizada que organizó al sistema educativo con base en la evaluación como tema central, particularmente en las últimas reformas educativas y con un fuerte sentido técnico que borra toda posibilidad educativa emancipadora, las propuestas escolares dirigidas a la niñez y juventudes indígenas mantienen inequidades estructurales. Estas van desde: a) condiciones materiales de las instituciones y de las comunidades; b) una oferta escolar marcada por un fuerte control sobre lo curricular para 'garantizar' aprendizajes comunes a todas/os, al mismo tiempo que -y bajo la noción de flexibilidad curricular- ha simulado márgenes inexistentes para ajustar el trabajo escolar de acuerdo con condiciones específicas, por ejemplo, aulas multigrado con presencia de más de dos lenguas; c) procesos formativos de los docentes, tanto de quienes laboran en escuelas del medio indígenas como de quienes se encuentran en las del sistema general, los que requieren de múltiples herramientas para el trabajo con la diversidad cultural y lingüística; d) la negación de las lenguas originarias como lenguas legítimas para aprender y producir conocimientos, y la simulación de un trabajo bilingüe (lengua indígena-español) para el trabajo escolar; e) el ocultamiento de las adscripciones comunitarias como efecto de la discriminación y racismos históricos, procesos que afectan directamente en los aprendizajes.

El avance en el tema de los derechos indígenas -expresado en el cambio al Artículo 2o. de la Constitución Política de los Estados Unidos Mexicanos (CPEUM) (2001)- estableció un escenario jurídico para operar cambios en un país que se reconoce como pluricultural y plurilingüe. Sin embargo, la concepción del 'otro' mantiene una posición de sujeto como otro 'vulnerable' a quien hay que atender en términos de políticas públicas. Es decir, salir de la concepción educativa intercultural compensatoria: aunque tengamos cambios en las leyes para el reconocimiento de los derechos de los pueblos, así como para la no discriminación, amparados en estatutos nacionales e internacionales, es un discurso con distancia de las prácticas. La idea de diversidad cultural sigue fetichizada (Zizek, 1998), y al mismo tiempo oculta la profundización de las desigualdades en contextos de capitalismo y neoliberalismo salvajes.

Con los cambios recientes en la Ley General de Educación (2019), se considera la interculturalidad como uno de los criterios transversales para todo el sistema educativo nacional, aspecto que no aparecía anteriormente, y se señala que con esta perspectiva se "promoverá la convivencia armónica entre personas y comunidades, sobre la base del respeto a sus diferentes concepciones, opiniones, tradiciones, costumbres y modos de vida y del reconocimiento de sus derechos en un marco de inclusión social" (Art. 16, frac. VIII). Lo anterior no se expresa aún en virajes significativos en todo el sistema, no obstante, en esa definición también se advierten combinaciones entre perspectivas de diversidad cultural e inclusión social en clave funcional.

Las deudas en el sistema educativo para con las comunidades y las y los sujetos indígenas tienen diversos aspectos que cruzan y hacen de cada contexto escolar y comunitario una realidad específica. Sin embargo, y aun frente a la 
multiplicidad de expresiones sobre la pertenencia a una comunidad denominada desde el Estado como indígena, el tratamiento de las culturas y de las lenguas originarias mantiene una orientación dicotómica.

A continuación, nos detendremos en dos dimensiones que funcionan como emblemas y dicotomías en las políticas de EIB impulsadas por el Estado que refieren: a un modo estático y esencialista de concebir a la cultura y la lengua de sujetos/comunidades indígenas para definirlos en sus identidades desde afuera; y a los enfoques sobre bilingüismo aplicados a lo escolar para comprender realidades multilingües complejas.

\section{La lengua y la cultura como emblemas de marcaciones identi- tarias en las políticas de EIB}

Para una mejor comprensión de los procesos escolares atravesados en distinta medida por las políticas de EIB, recuperamos algunas de las perspectivas que expresan jóvenes indígenas al referirse a sus comunidades y modos de autopercibirse, las que suelen ser muy diferentes a los modos a través de los cuales desde las políticas públicas -en este tema, las educativas- se refieren a ellos. Por ejemplo, y como parte del trabajo formativo y de diálogo con estudiantes de la licenciatura de educación indígena de la UPN, es muy frecuente escuchar a las y los jóvenes indígenas comentar cuestiones que refieren a que su conocimiento sobre la denominación de 'indígenas' -como forma de nombrarlos desde la academia y las políticas públicas- ocurre cuando salen de las comunidades y en muchos casos cuando llegan a la universidad (Czarny, 2012). Esta forma de autorreconocerse no tiene como sustento una definición de sujeto o comunidad a través de emblematizar 'lo cultural' y 'la lengua' del grupo en cuestión, símbolos utilizados desde el Estado para construir las políticas indigenistas denominadas interculturales y bilingües. Más bien, en la expresión de las y los jóvenes se recupera la idea de ser una otredad marcada por el concepto de 'indígena' el que se advierte estando 'afuera' de la comunidad. Elena Yasnaya Aguilar, lingüista mixe, comenta:

La conciencia de ser indígena me nació cuando llegué a la ciudad, aprendí que lo era y me percaté de sus implicaciones. La primera vez que le conté a mi abuela (monolingüe en mixe) sobre el hecho de que ella, al igual que yo, era indígena, lo negó. Ella es mixe, no indígena. (Aguilar, 2017, p. 19)

Al mismo tiempo, los jóvenes se reconocen con modos propios para autodefinirse, usando los gentilicios de sus pueblos originarios en sus propias lenguas, por ejemplo: ñuu savi (el pueblo de la lluvia), los ayuujk jä̈'y (gente de la lengua de las montañas); ikoots (nosotros), entre otras. El uso de las lenguas originarias para autoadscribirse a un pueblo es un acto de negación de la categoría externa de 'indígena' y, por tanto, un acto de resistencia. Aguilar menciona al respecto: "soy indígena en la medida en que pertenezco a una nación encapsulada dentro de un Estado que ha combatido, y combate aún, la existencia misma de mi pueblo y de mi lengua, que niega la historia de mi pueblo en las aulas" (Aguilar, 2017, p. 21).

También en ese autonombrarse se encuentran formas como "nosotros los del pueblo San Jerónimo", o, "somos del pueblito de San Isidro", entre miles de otras referencias, haciendo explícito un tipo de pertenencia al actual territorio en el que se habita pero bajo la denominación oficial dada por las circunscripciones estatales. En su análisis sobre identidades en el campo de la 
antropología, que fue central entre las décadas de 1980 y 1990, Bartolomé (1997) indicaba que existen diversos modos de abordar la pertenencia comunitaria, que pueden ser desde las denominadas identidades étnicas, estratégicas, residenciales hasta las panindias. Asimismo, en el debate contemporáneo, por ejemplo, en el tema de juventudes indígenas, Urteaga y Cruz (2020) plantean una agenda epistémica para la investigación con las diversidades y el trabajo de la categoría jóvenes indígenas, y señalan la importancia de que reconozcamos en la investigación los propios modos de nombrarse que tienen las y los sujetos para salir de categorías externas que siguen racializando a diversos sectores, en este caso, históricamente subalternizados.

No obstante, en las políticas de EIB, la permanente referencia a un ellos genérico 'los indígenas' postula que todos los así denominados deberían identificarse del mismo modo y bajo los mismos atributos y condiciones. El hecho de que no se reconozca como perteneciente a alguno de los marcadores identitarios establecidos principalmente por vinculación a la lengua indígena y/o a una referencia a su cultura comunitaria los ubicaría como 'perdidos' o 'aculturados' de sus pueblos. Lo anterior resulta indicativo, por ejemplo, en las políticas públicas de acción afirmativa para indígenas, las que generalmente solicitan una muestra de pertenencia a un pueblo o comunidad, y ello se realiza a partir de constatar el ser hablante de una lengua originaria.

Así también, el acento desde los enfoques de EIB de las políticas educativas para indígenas, al resaltar su existencia como pueblos originarios por 'su cultura' y 'su lengua indígena' -no por reconocer territorios y recursos naturales que son extraíbles para fines otros- ha generado, en algunos casos, una profundización de la diferencia, en lo que se busca obstinadamente marcar como 'dos culturas'. Lo anterior se advierte en las definiciones que realizan maestros indígenas que laboran en escuelas pertenecientes al subsistema escolar DGEIIB. Por ejemplo, en una escuela de los Altos de Chiapas, en el municipio de San Juan Cancuc, donde casi el 95\% de los pobladores son hablantes de tseltal como lengua materna; un maestro expresaba lo siguiente sobre la EIB:

Bueno, respecto a eso yo entiendo que la educación intercultural es cuando hay diferentes grupos culturales en un mismo espacio. Bueno aquí la cultura que predomina es el tseltal hay padres de familia e incluso niños que desde sus familias aprenden español. Aquí se trata de que la cultura tseltal como la cultura mestiza conviva con respeto. Y por lo tanto debemos enseñarles a los niños las particularidades de la cultura mestiza. Hay que reforzar en ellos lo valioso que es ser indígenas, de ser hablantes de una lengua y que no pasa nada, también se puede convivir con la gente mestiza y con los demás pueblos [...] Bueno, personalmente creo que es un sistema que debería equilibrar ambas culturas [...]. (testimonio, en Sántiz, 2018, pp. 104-105)

Esta expresión muestra la persistente dicotomía entre un 'ellos' (mestizos) y un 'nosotros' (tseltales); lo anterior, derivado de una concepción de las políticas de EIB de acuerdo con la cual lo que los docentes entienden que deben hacer es revalorizar la cultura propia, en este caso la tseltal, y la otra cultura, que es la mestiza. Al mismo tiempo, la forma en que se ha buscado establecer límites entre una cultura y otra para 'darle lugar', en este ejemplo, a la cultura tseltal, no solo anula la complejidad cultural, política y social que hay en 'lo mestizo' y en 'lo indígena', sino que muestra la imbricación de lengua y cultura -ser de la cultura tseltal porque se habla en tseltal- como elementos constitutivos e indivisibles para plantear una educación intercultural bilingüe. Deja al descubierto que, y aun con algunos esfuerzos, la posición de revalorar la cultura 
maya-tseltal sigue siendo un ejercicio difícil de realizar en la escuela. En ese mismo contexto de trabajo escolar, cuando se les pregunta a los padres y madres de familia sobre la valoración de la escuela para sus hijos, reconocen que hay mayor presencia de la lengua tseltal, por ejemplo, en las reuniones escolares a las que asisten los adultos progenitores, o en las conversaciones entre maestros y niñas y niños; no obstante, la enseñanza sigue llevándose adelante en español. Recuperando el trabajo de esta investigadora y docente de escuelas indígena, Reyna Isabel Sántiz (2018), perteneciente a pueblos mayas-tseltales y hablante del tseltal y del español, señala que para conversar con la gente en la comunidad no se puede preguntar a través de la referencia de cómo ven o entienden la educación intercultural bilingüe, concepto que no solo no existe como expresión en la lengua sino que no cobra valor en la comunidad. En su perspectiva señala que "es necesario una educación donde no se anteponga una realidad sobre otra, [...] Una propuesta que conjunte las dos realidades las de los tseltaletik [hablantes de tseltal] y los no tseltaletik, para superar lo estático de los modelos educativos actuales" (Sántiz, 2018, p. 88). Además, documenta otros sentidos que tiene el usar la lengua originaria en la educación como miembros activos en sus comunidades:

Los hombres y mujeres tzeltales decidimos lo que deseamos y queremos de acuerdo con el "Lekilk'uxlejal” [buena vida], la cual está basada en la sabiduría, la razón y la convivencia armónica. Aún es posible observar las prácticas culturales de educación, las cuales se configuran en diversos espacios, como la casa, la familia, la comunidad, la milpa, la naturaleza misma, así como su organización social en clanes y linajes, su sistemas de cargos, el uso del calendario, y sobre todo en el uso de la lengua tseltal, la cual es el medio de comunicación que utilizan para transmitir todos los conocimientos, acontecimientos y sentimientos que surgen en la comunidad. (Sántiz, 2018 p. 163)

Hay una inmensa distancia entre, por un lado, los modos en que comunidades como esta maya-tseltal entienden los valores y las prácticas culturales que se configuran en diversos espacios y, por otro, los discursos educativos interculturales y bilingües que se proponen para escolarizaciones de comunidades originarias. Así también, el discurso de revalorar la cultura y la lengua de los denominados indígenas por parte de las políticas tiene poca credibilidad frente a prácticas continuas de castellanización en las escuelas primarias indígenas. Solo para cerrar este apartado nos interesa señalar que también desde las políticas interculturales se culpa a los docentes indígenas por no usar sus lenguas originarias en los procesos escolares; y más allá de lo que en cada contexto le implique al docente usar y enseñar en la lengua, el reclamo desde arriba sigue marcando -ahora en versión de acción afirmativa sobre esta diferencia- una tutela sobre los pueblos.

\section{Las dicotomías en los enfoques bilingües de la EIB}

La característica 'bilingüe' ha estado presente en la educación dirigida a las poblaciones indígenas desde al menos la década de 1930. Primero como política de asimilación, y después, como forma de incorporar a estas poblaciones a la sociedad nacional, para que tuvieran acceso a las instituciones de salud, escolares y económicas estatales. En la década de 1980, se agregó la característica de bicultural y después de más de 70 años en la EIB, sigue como característica esencial de esta.

Desde la EIB, la noción de bilingüismo, según Zavala, está dominada por "ideas monoglósicas que asumen las lenguas como sistemas completos y delimitados" 
(2018, p. 58). A partir de ahí se asume la existencia de una diglosia entre el español y una lengua indígena, es decir que los vínculos entre ambas lenguas están enmarcadas en una relación vertical de valoración. En este caso, el español es la lengua hegemónica que se impone en su uso a las lenguas indígenas por el hecho de ser valorada como una lengua con mayor valor de uso y prestigio. Por tanto, las actividades escolares tienen que ir encaminadas a construir un bilingüismo equilibrado que rompa esa relación de discriminación y construya sus equilibrios de ambas lenguas, y que las personas que son bilingües tengan las cuatro competencias lingüísticas en ambas lenguas: escribir, hablar, leer y entender oralmente.

Sin embargo, en México, a más de cuarenta años de políticas explícitas de bilingüismo en educación básica, esto no se ha conseguido. Algunas razones que identificamos son las siguientes: 1) la lengua se enseña como si fuera un contenido escolar, una disciplina, con horario fijo para su uso y aprendizaje; aunque desde 2008 se tiene el enfoque de 'prácticas sociales del lenguaje', al convertirlo en un contenido escolar se rompe con la noción básica de que el lenguaje se utiliza para hacer cosas en el mundo y con personas (Zavala, 2018). 2) Se tiene una noción estructural del lenguaje que mira las competencias lingüísticas en módulos independientes, separados, por lo tanto, para romper las interferencias se debe de enseñar de forma separada, lo que imposibilita el uso continuo de ambas lenguas. 3) La perspectiva no permite ver otros repertorios lingüísticos en contextos que son multilingües. 4) La ubicación de maestros/as fuera de su zona lingüística por acuerdos sindicales, al igual que el uso de libros de texto que no son de las variantes locales. Y por último, como ya mencionamos anteriormente, la no continuación del subsistema de educación indígena de primaria a secundaria, instancia en la que se arraigan las cuatro competencias lingüísticas del español y, por tanto, sería el espacio idóneo para el uso continuo de las lenguas locales.

Aunado a esto, la conceptualización de que lengua se relaciona unívocamente con cultura e identidad -que hemos desarrollado a lo largo de este texto- termina, por un lado, folclorizando los usos de la lengua y fijándolos a prácticas escolares como saludos o bienvenida a autoridades y vistas, canto de himno nacional en lengua indígena, traducciones de textos, etc. Por otra parte, deja de lado procesos sociales en los que la educación y los niños y niñas y jóvenes están inmersos, como la migración y el uso de redes sociales.

Esta perspectiva dicotómica de bilingüismo se pone en cuestión cuando analizamos prácticas educativas en las que los jóvenes utilizan diferentes lenguas para resolver una sola actividad escolar. Por ejemplo, en el caso de un modelo de educación secundaria comunitaria indígena en el estado de Oaxaca (Secundaria Comunitarias Indígenas) donde se utiliza la entrevista a los abuelos/sabios de la comunidad como forma de indagar el conocimiento local comunitario, los estudiantes construyen un guion de entrevista en su lengua indígena. Al momento de hacer la entrevista, algunos de los estudiantes realizan las preguntas al abuelo/a y los demás van apuntando en sus cuadernos.

Los apuntes de los estudiantes están escritos en español, lo que significa que, al estar escuchando los relatos de los abuelos/as, hacen una traducción simultánea y utilizan los recursos lingüísticos que tienen en escritura, en español. En la siguiente fotografía se muestran los apuntes de una entrevista de un estudiante de primero de secundaria (séptimo de educación fundamental/básica): 
Fotografía 1. Anotaciones de un estudiante de $1^{\circ}$ de secundaria al hacer una entrevista.

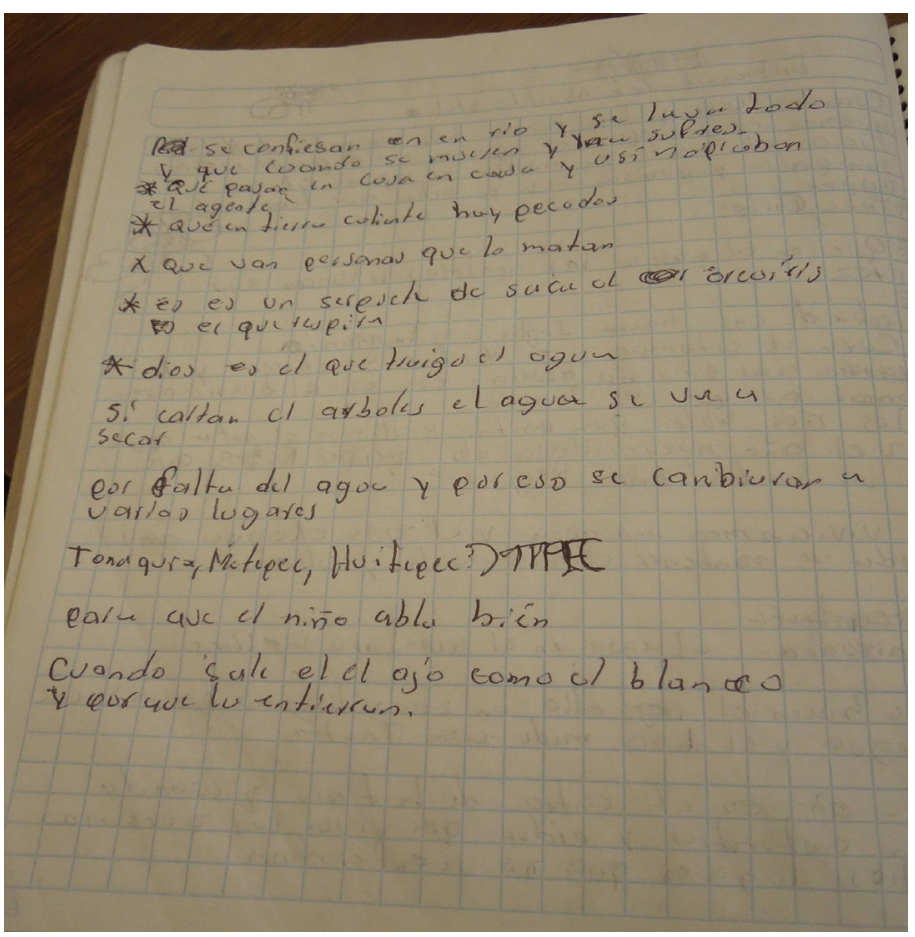

En la imagen se observa que su información está muy bien organizada y, al compararlo con respecto a las preguntas del guion de entrevista, cada una de ella es una respuesta a una pregunta determinada. En relación con el contenido de los apuntes, pareciera que el estudiante utiliza palabras clave para anotar lo que el portador está comentando, técnica importante dentro de la metodología de entrevistas. Es decir, registrar información suficiente que después aportará para la construcción de otros textos. Aunque se pueden observar dificultades de ortografía en español, el estudiante tiene recursos suficientes para resolver una tarea en una situación multilingüe, escucha en su lengua local y escribe en español. Jørgenson, Karrebæk, Madsen y Møller (2011) proponen que, en situaciones multilingües, las personas utilizan recursos lingüísticos que están a su disposición para lograr sus objetivos comunicativos de la mejor forma posible. Para comprender esta actividad es necesario dejar de ver dos sistemas lingüísticos en cajones separados de nuestra cognición y ver más bien como un único sistema semiótico integrado por diversos léxicos y gramáticas verbales y no verbales.

Las perspectivas críticas permiten ver la utilización de diferentes repertorios lingüísticos para insertarse, dar sentido y comprensión a cierta práctica comunicativa (Heller, 2007; García, 2009; Blommaert y Rampton, 2011; Canagarajah, 2011). Las interacciones entre los usos de lenguas no se ven como subordinaciones o code-switching, sino como repertorios que se utilizan dentro de una práctica social con fines comunicativos; como sistemas integrados y no como dos lenguas separadas con lógicas diferentes (Canagarajah, 2011; García y Lin, 2016; Zavala, 2018). Por lo tanto, se cuestiona la misma idea del bilingüismo, pues las personas tenemos repertorios lingüísticos de más de una lengua. 
Ofelia García (2009) propone usar el concepto de 'translingüismo' para poner énfasis en los recursos dinámicos y movibles que las personas adoptan a situaciones comunicativas específicas. De tal forma que el foco no son las lenguas, sino las prácticas de las personas con respecto a esas lenguas.

En este mismo caso de las Secundarias Comunitarias Indígenas, el uso continuo y flexible de la lengua local/indígena tanto de forma oral como escrita (Briseño-Roa, 2020) rompe con la idea de la disciplinarización de la lengua que se plantea en los planes y programas nacionales referidos a la EIB. Los resultados conseguidos son que los estudiantes desarrollen repertorios lingüísticos en dos lenguas, la local y el español:

\begin{abstract}
He visto como estudiantes que entraron de 12 años con habilidades de lectura en español poco desarrolladas así como sin haber escrito en mixe ni una palabra, terminaron la Secundaria teniendo una escritura fluida en ambas lenguas y una comprensión lectora en ambas con el nivel esperado. (Briseño-Roa, 2018)
\end{abstract}

Mirar los repertorios que tienen y usan los niños y las niñas en contextos donde conviven dos o más lenguas significa dejar de identificarlos/as como deficientes lingüísticamente, más bien supone que se les reconozca como usuarios de un amplio rango de repertorios lingüísticos. Adoptar esta perspectiva permite ver las competencias que de otras formas no son válidas en los ambientes escolares y que se inserten en prácticas que los sitúen como sujetos activos. Y por tanto, deja en evidencia que las lenguas subalternizadas no son un lastre para el aprendizaje del español, es decir, el desplazamiento no es lingüístico sino es social, es estructural y es colonial.

\title{
A modo de cierre
}

En la revisión realizada en este texto buscamos mostrar que la perspectiva escolar dirigida a las poblaciones indígenas muestra una continuidad de las políticas indigenistas aún y bajo las propuestas de EIB, tanto por la invisibilización de la heterogeneidad de la población a la que se dirige, como por la rigidez de entendimiento hacia procesos complejos entre cultura, lengua e identidad. Sin embargo, aunque se ha avanzado en 'sensibilizar' sobre la(s) diversidad(es), aún no es el camino para que el 'chaleco de fuerza de la institución escolar' habilite otras prácticas.

A más de cincuenta años de propuestas educativas denominadas interculturales bilingües, cabe preguntarnos si han logrado transformar las políticas y prácticas de vida en nuestros estados y sociedades. Retomando a López (2019), es necesario identificar si la evocación de la interculturalidad "va gradualmente transfigurando el sentido de lo que el imaginario nacional concibe como cultura nacional, así como el propio significado de lo nacional, en relación con la opresión cultural y la explotación económica de las poblaciones originarias" (2019, p. 49). Consideramos que esta educación intercultural bilingüe, así como la interculturalidad en tanto perspectiva impulsada desde las políticas de Estado, no ha logrado responder a procesos sociales complejos que se están viviendo en el siglo XXI, como la migración, las diversas formas de reconocerse como miembro de una comunidad, las diversidades de repertorios lingüísticos presentes en espacios escolares, entre otros. Los procesos identitarios van más allá de categorías dicotómicas y de las delimitaciones espaciales que se buscan imponer desde los marcos de políticas públicas. Es necesario retomar perspectivas interseccionales para comprender estas complejidades. 
La forma de definir a las/los sujetos y comunidades, marcados por una diferencia en las historias nacionales, ha configurado un vasto debate político y epistémico en las academias con sus distintas posiciones, el que orienta discursos y contradiscursos sobre la composición de un 'nos-otros' que es más bien un 'sin-otros' y 'sin-otrxs'. Estas alteridades que se construyeron junto con la idea de Estado-nación en las políticas interculturales continúan silenciando a los otrxs, sus formas de vida y de organización, lo que para estas poblaciones significa no solo el no acceso a la justicia social, sino destrucción y violencia subjetiva, cultural, territorial y física.

Probablemente, el imaginario nacional de lo que se concibe como cultura nacional es de los puntos más complejos para el análisis en los debates denominados interculturales (López, 2019). Sin embargo, lo que observamos en el campo educativo y a partir de la irrupción de los movimientos sociales indígenas de las últimas décadas y el uso, en distinta medida, de propuestas y demandas en algunos escenarios, bajo la denominación de interculturalidad e intraculturalidad, entre otros, es la disputa por el sentido de la escuela y los proyectos políticos que con ella se conforman. El discurso de la interculturalidad resultó un arma para la visibilización de los procesos escolares de exclusión y discriminación que han enfrentado las comunidades indígenas, discurso que diversas organizaciones etnopolíticas han retomado para plantear sus propias definiciones y demandas.

Entre los temas pendientes, sigue allí la pregunta de qué escuela y para qué aprendizajes. La escuela (escolarizarse) como acto político implica más que diseños curriculares y modelos educativos que se denominen como interculturales y bilingües. Indudablemente, se han explorado enfoques pedagógicos más cercanos y potentes para dar cabida a las diversidades y procesos de identificación 'pluri', pero siguen pendientes los derechos y las justicias culturales, epistémicas, así como la participación directa y toma de decisiones de las comunidades y pueblos en el tipo de proyecto educativo escolar en el que quieren estar, así como en distintos ámbitos de la vida social.

Reconocemos la existencia de proyectos alternativos que desde organizaciones sociales y magisteriales han construido una educación que rompe algunas de las dicotomías planteadas, por ejemplo, la posibilidad de trabajar contenidos locales/comunitarios dentro de la escuela y un uso simultáneo de las lenguas en distintos momentos. En varias latitudes hay una búsqueda por recuperar epistemes de los pueblos bajo perspectivas denominadas decoloniales, algunas en el formato de la institución escolar y otras, buscando formatos diversos.

Para finalizar, resulta importante aclarar que este texto se escribe en tiempos aún de pandemia por COVID-19 y, como se ha documentado en diferentes ámbitos tanto en redes y webinarios como en materiales escritos, la profundización de las inequidades y desigualdades por 'acceder' a la escuela vía plataformas, dispositivos y medios televisivos ha alejado aún más a muchas niñas, niños y jóvenes indígenas. El reto por el derecho al acceso a la escuela parece nuevamente quedar atrapado, tema sobre el cual se nos presentan nuevos desafíos. 


\section{Q Referencias bibliográficas}

Aguilar, Y. (2017). ËËts, ATOM. Algunos apuntes sobre la identidad indígena. Revista Universidad, sept., 17-23.

" Bartolomé, M. (1997). Gente de costumbre y gente de razón. Las identidades étnicas en México. México: Siglo XXI.

» Blommaert, J. y Rampton, B. (2011). Language and Superdiversity. Diversities, 13(2), 1- 20.

» Bonfil, G. (1991). Pensar nuestra cultura. México: Alianza.

» Brayboy, B. y Maughan, E. (2009). Indigenous Knowledge and the story of the bean. Harvard Educational Review, 79(1), 1-21.

"Briseño-Roa, J. (2018). Cultura escolar comunitaria: prácticas, textos y voces de las Secundarias Comunitarias Indígenas del Estado de Oaxaca (Tesis de doctorado). Centro de Investigación y de Estudios Avanzados del Instituto Politécnico Nacional (Cinvestav), México.

》 Briseño-Roa, J. (2020). La construcción cotidiana de un modelo pedagógico alternativo en educación indígena: entre la participación comunitaria y el uso continuo de la lengua indígena. De Prácticas y Discursos. Cuaderno de Ciencias Sociales, 9(13). http://dx.doi. org/10.30972/dpd.9134411

" Canagarajah, S.(2011). Translanguaging in the classroom: emerging issues for research and pedagog. Applied Linguistics Review, 2, 1-28.

"Czarny Krischkautzky, G. (2012). Jóvenes indígenas en la UPN. Relatos desde la educación superior. México: UPN.

"Czarny, G. (2017). Schooling Processes and the Indigenous Peoples in Urban Contexts in Mexico. En W. Pink y G. Noblit (Eds.). Second International Handbook of urban education, vol. 1 (pp. 487-505). Suiza: Springer.

» Czarny, G. y Salinas, G. (2020). Antes, durante y ¿después? del Covid: desigualdades, inequidades y discriminación en la educación escolar indígena. En I. Dussel, P. Ferrante y D. Pulfer (Coords.). Pensar la educación en tiempos de pandemia Il. Experiencias y problemáticas en Iberoamérica (pp. 145-156). Ciudad de Buenos Aires: CLACSO-UNIPE.

"DGEI (2008). Parámetros Curriculares de la Lengua Indígena. México: Secretaría de Educación Pública.

" Díaz-Couder, E. (2000). Diversidad sociocultural y educación en México. En S. Comboni y J. Juárez (Eds.). Globalización, educación y cultura (pp. 105-148). México: Universidad Autónoma de México.

"Fondo de las Naciones Unidas para la Infancia (UNICEF) e Instituto Nacional para la Evaluación Educativa (INEE). (2018). Panorama educativo de la población indígena y afrodescendiente 2017. México: UNICEF/INEE.

» García, O. (2009). Bilingual Education in the 21st Century: global perspectives. Malden: Willey-Blackwell.

» García, O. y Lin, A. (2016). Translanguaging in bilingual education. En O. García, A. Lin y S. May (Eds.). Encyclopedia of Language and Education, Vol. 5. Bilingual Education (pp. 117-130). Dordrecht: Springer.

》 Heller, M. (Ed.) (2007). Bilingualism a social approach. Reino Unido: Palgrave McMillan. 
" Hernández, R., Sieder, R. y Sierra, T. (Eds.) (2013). Introducción. En Justicias indígenas y Estado. Violencias contemporáneas (pp. 13-47). México: FLACSO-CIESAS.

» Jiménez, Y. (2009). Cultura comunitaria y escuela intercultural. México: Coordinación General de Educación Intercultural y Bilingüe.

» Jørgenson, N., Karrebæk, M., Madsen, L. y Møller, J. (2011). Polylanguaging in Superdiversity. Diversities, 13(2), 22-38.

» López, L. (2019). Interculturalidad y políticas públicas en América Latina. En J. González (Ed.). Multiculturalismo e interculturalidad en las Américas. Canadá, México, Guatemala, Colombia, Bolivia, Brasil, Uruguay (pp. 46-101). Bogotá: Cátedra UNESCO-Diálogo intercultural, Universidad Nacional de Colombia.

" López-Caballero, P. (2015) Las políticas indigenistas y la 'fábrica' de su sujeto de intervención en la creación del primer Centro Coordinador del Instituto Nacional Indigenista (1948-1952). En D. Gleizar y P. López Caballero (Coords.), Nación y alteridad Mestizos, indígenas y extranjeros en el proceso de formación nacional (pp. 69-108). México: Universidad Autónoma de México.

» Martínez, R. (2007). Vivir invisibles: la resignificación cultural entre los otomíes urbanos de Guadalajara. México: Publicaciones de la Casa Chata, CIESAS.

» Mato, D. (2018). Educación superior y pueblos indígenas y afrodescendientes en América Latina: aspectos sociales y normativos, experiencias, logros, aprendizajes y desafíos. En D. Mato (Coord.). Educación superior, diversidad cultural e interculturalidad en América Latina (pp. 15-37). Córdoba: Universidad Nacional de Córdoba.

"Navia, C., Czarny, G. y Salinas, G. (2020). Marcas étnicas y auto-reconocimiento de Estudiantes indígenas en educación Superior. Archivos Analíticos de Políticas Educativas (EPAA), 28(166). DOl.14507/epaa.28.4781

»Pérez-Ruiz, M. (2016). La traducción y la hibridación como problemas para una interculturalidad autónoma, colaborativa y descolonizadora. Liminar, XIV(1), 15-29.

"Quijano, A. (2000). Colonialidad del poder, eurocentrismo y América Latina. En E. Lander (Comp.). La colonialidad del saber: eurocentrismo y ciencias sociales. Perspectivas latinoamericanas (pp. 201-246). Buenos Aires: CLACSO.

»Rufer, M. (2012). El habla, la escucha y la escritura. Subalternidad y horizontalidad desde la crítica poscolonial. En S. Corona y O. Kaltmeier. En diálogo. Metodologías horizontales en Ciencias Sociales y Culturales (pp. 55-84). Barcelona: Gedisa.

» Rockwell, E. y Anderson-Levitt, K. (2017). Introduction. En K. Anderson-Levitt y E. Rockwell (Eds.). Comparing ethnographies: Local studies of education across the Americas, (126), Washington, DC: American Educational Research Association,

"Rockwell, E. y González, E. (2016). Antropología de los procesos educativos en México 1995-2009. Cuadernos del Sur, 21(41), 6-30.

» Sántiz, R. (2018). ¿De qué educación intercultural y bilingüe hablamos? Prácticas docentes en una escuela de educación indígena. Chiloljá, San Juan Cancuc, Chiapas (tesis de Maestría). Universidad Pedagógica Nacional, Ajusco.

"Segato, R. (2007). La nación y sus otros. Raza, etnicidad y diversidad religiosa en tiempos de Políticas de la Identidad. Buenos Aires: Prometeo.

» Secretaría de Educación Pública (SEP) (2017). Aprendizajes clave para la Educación Integral. Plan y programas de estudio para la Educación Básica. México: SEP.

» Spivak, G. (2003) ¿Puede hablar el subalterno? Revista Colombiana de Antropología, 39, 297-364. 
" Tubino, F. (2004). Del interculturalismo funcional al interculturalismo crítico. En M. Samaniego y C. Garbarini (Comps.). Rostros y fronteras de la identidad (pp. 151-165). Temuco: Universidad Católica de Temuco.

»Urteaga, M. y Cruz, T. (Coords.) (2020). Estudios sobre las juventudes indígenas. Hacia una epistemología de lo juvenil étnico. En Juventudes indígenas en México. Estudios y escenarios socioculturales (pp. 23-50). México: ECOSUR, UNICACH, CESMECA, ENAH.

»Walsh, C (2009). Interculturalidad crítica y pedagogía de-colonial: insurgir, re-existir y re-vivir. En P. Medina (coord.). Educación intercultural en América Latina. Memorias, horizontes teóricos y disputas políticas (pp. 27-54). México: UPN, CONACYT, Plaza y Valdés.

"Zavala, V. (2018). El lenguaje como práctica social: cuestionando dicotomías y esencialismos en la educación intercultural bilingüe. Sílex, 8(1), 57-76.

»Zizek, S. (1998). Multiculturalismo o la lógica cultural del capitalismo multinacional. En Estudios Culturales. Reflexiones sobre el multiculturalismo (pp. 137-188). Buenos Aires: Paidós/Biblioteca Virtual de Ciencias Sociales. Recuperado de: http://www.cholonautas. edu.pe/busca.php?busca=zizek

»Otras fuentes consultadas

»Constitución Política de los Estados Unidos Mexicanos. (2001, 14 de agosto). Reforma al Artículo Segundo. México: DOF.

» Ley General de Educación (2019). Decreto por el que se expide la Ley General de Educación y se abroga la Ley General de la Infraestructura Física Educativa. México: DOF, 30/09/2019.

》SEP (2019). Acuerdo número 20/11/19 por el que se modifica el diverso 12/10/17 por el que se establece el plan y los programas de estudio para la educación básica: aprendizajes clave para la educación integral. México: DOF. 
